\title{
SNS BEAM CHOPPING AND ITS IMPLICATIONS FOR MACHINE PROTECTION*
}

\author{
Coles Sibley, ORNL, Oak Ridge, TN 37831, USA \\ Larry Doolittle, LBNL, Berkeley, CA 94720, USA
}

\begin{abstract}
The SNS front end includes a high speed chopping system, which has the primary purpose of creating the $250 \mathrm{~ns}$ beam gaps essential for safe ring kicker operation. This system is also predicted to be capable of extinguishing the beam completely, and holding it off indefinitely, without damaging or activating any hardware, and without even changing the thermal equilibrium of any RF gear. It is, however, an intrinsically non-fail-safe approach to blanking an $\mathrm{H}^{-}$beam of up to $2 \mathrm{MW}$ average power. This paper will describe the front end chopping process, and the interlock systems that may allow it to be used as a convenient initial mechanism for on-demand beam blanking. It will also describe the instrumentation that can detect faults and initiate slower, more reliable, but operationally less desirable means of turning off the beam.
\end{abstract}

\section{MACHINE PROTECTION SYSTEM}

The SNS Machine Protection System[2] is designed to provide defense in depth against damage to machine components caused by mis-steered beam or component failure. It will also facilitate tuning by shortening the beam pulse when losses are high, minimizing radiation according to ALARA. The system is a combination of software applications, fast beam loss detection, system failure detection, and a high QA hardwired protection system. The QA of each part of the subsystem depends on the probability of failure, consequence of failure and machine availability. For instance, software is allowed to change some trip levels for ease in commissioning, while other devices in the hardwired system cannot be bypassed through software. There are two types of shutdown scenarios: latched shutdowns, which require an operator to acknowledge the fault and reset it, and shutdowns that will automatically be reset for the next beam pulse. Both types of shutdowns will use the Front End choppers, to take advantage of their fast response time.

Non-latching shutdown commands from Beam Loss Monitors are likely to be a routine part of machine tuning. So while it is important to kill the beam reliably in this case, it is also desirable to make the shutdown mechanism as minimally invasive as possible, so the machine's operability on the next pulse will not be jeopardized. This implies that neither cavity RF (including the RFQ) nor the source plasma should be turned off unnecessarily.

\footnotetext{
${ }^{*}$ Work supported by the Director, Office of Science, Office of Basic Energy Sciences, of the U.S. Department of Energy under Contract No. DE-AC03-76SF00098
}

As described below, the LEBT chopper can deflect at least $98 \%$ of the beam into the $65 \mathrm{keV}$ "dump". Because of the vagaries of this chopper, however, there is no possible way for it to be considered a fail-safe shutoff. Unless the MPS has a fast and reliable means of detecting its failure, an additional beam kill method must be activated, killing the RF drive to the RFQ.

The trick to effectively using this capability is to have fast and reliable means of detecting its failure, and to have a fast and reliable backup method to kill the beam. Fast in this case means the total time from primary shutdown input to beam shutdown, through the secondary channel, must not exceed $10 \mu \mathrm{s}$.

The RFQ provides a satisfactory means to shut down the beam reliably. A kill switch in the RF drive line will reduce the field to a non-propagating level in about $4 \mu$ s [1]. If the system resorts to this backup method, the fault shifts to the "latching" category, because the thermal equilibrium in the RFQ is disturbed. Figure 1 shows the architecture schematically.

\section{SECOND STAGE BEAM SHUTOFF}

The primary means of detecting failure of the LEBT chopper is to measure and fault-check eight signals at $32 \mathrm{MHz}$ : the four deflection electrode voltages, and the four split-electrode current signals. The process of digitizing waveforms of current and voltage is fail-safe: the chance that broken wires, changes in gain, and extraneous random noise can take an invalid signal and make it pass a long series of window tests is vanishingly small. All obvious hardware failure modes are detected by this arrangement. These include failures in the $\pm 3 \mathrm{kV}$ power supply, the high voltage switch, the capacitive coupling network, the timing generator, and cables between the above components. The split electrode measurement is designed to detect small amounts of mis-steering. It is also true that, by looking at the simple sum signal, mis-steering that is sufficient to let beam through during the intended beam-off times can also be detected.

During intended beam-off times, a badly focused beam at the LEBT chopper target can produce more current though the RFQ than can be safely handled in the rest of the accelerator. This is the only failure mode that can not be detected by analysis of the current and voltage waveforms in the LEBT chopper subsystem. Additional cross checks, based on BPM and BCM pickups in the MEBT, must be used to guard against this possibility. 


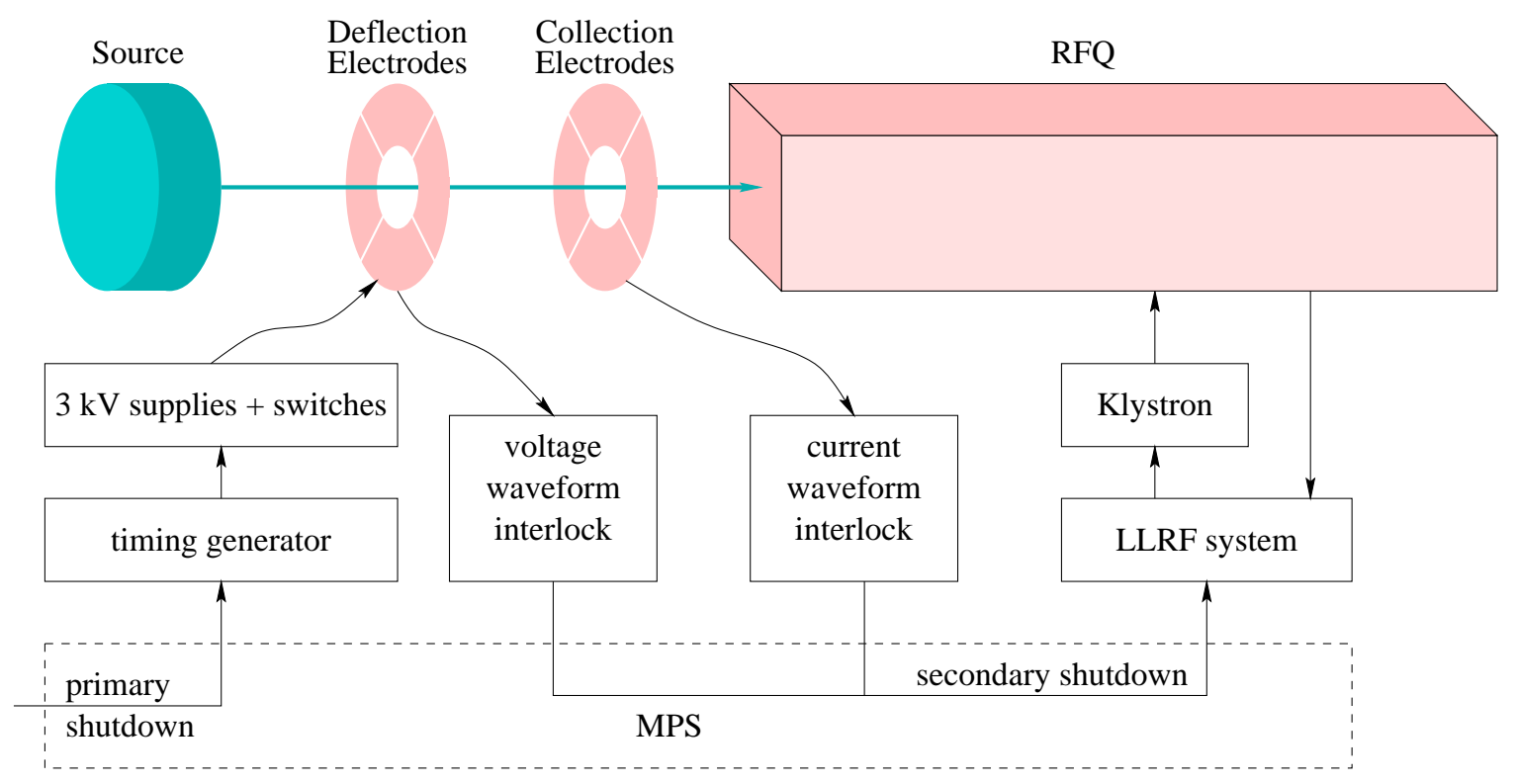

Figure 1: Schematic of the beamline and redundant cutoff mechanism.

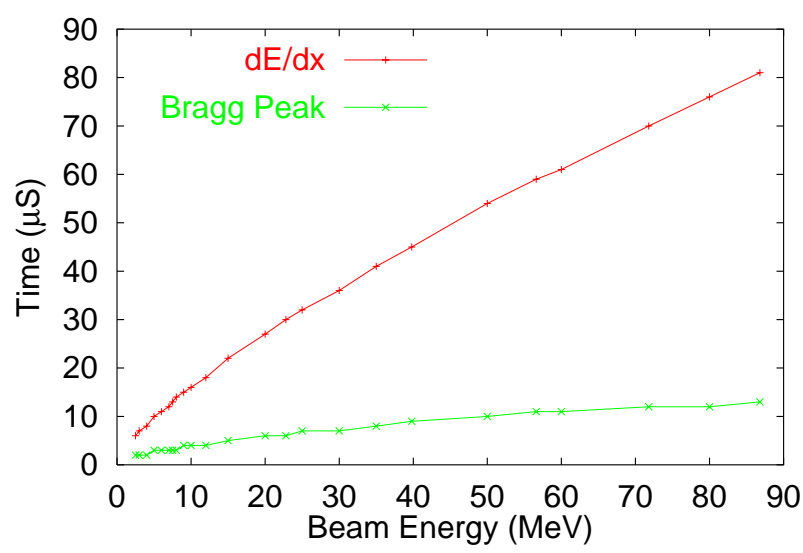

Figure 2: Copper survivability in the SNS.

\section{SPEED}

Beam loss can damage copper in at least two ways, too much average power lost over many pulses (Watts), or instantaneous beam loss or peak power (Joules or Joules per gram). The instantaneous damage is a function of time, beam size, peak current, and beam energy [3][4]. Calculations indicate the response time for the MPS, including detection of loss, MPS transmission delays, and beam shutdown range from $5 \mu \mathrm{s}$ in the DTL $(E<7.5 \mathrm{MeV})$ to $20 \mu \mathrm{s}$ at the end of the CCL $(E>87 \mathrm{MeV})$.

Analysis of digitized waveforms is needed within this time frame. Conventional computer technology (microcontroller, DSP, RISC, and multi-CPU collections of the above) may reach very high throughput and average speed, but their guaranteed latency behavior is not so good. The current generation of FPGA chips can, when carefully used, cycle at 50 to $200 \mathrm{MHz}$, and perform simple computa- tions with a few cycles of latency. Parallel, adjustable window comparisons of four to eight channels count as simple computations.

FPGA chips are uniquely testable, suggesting that they have a place in a high QA system. All logic circuits and wires on the chip are fully tested at the factory. Within limits, the firmware that runs the FPGA can be tested in a software simulator (this is one of the big reasons that use of Verilog and VHDL is so widespread). With the addition of bench-test design verification, using such time honored methods as temperature and frequency guard band testing, we expect to meet standards of sound engineering suitable for the project's QA-2 level.

\section{LEBT CHOPPER OPERATION}

LEBT Chopper operation is explained in more detail elsewhere [5]. High speed chopping waveforms are capacitively coupled onto electrodes whose D.C. potential sets the static steering and focusing behavior. Because of that A.C. coupling, the chopping waveforms have to be designed to have zero D.C. component. The waveforms shown in figure 3 have that property. The only time a small amount of beam might leak through the chopper is during the $40 \mathrm{~ns}$ transitions between dwell points, when the deflection is $1 / \sqrt{2}$ of the design point. These events happen at roughly $1 \mathrm{MHz}$, suggesting that the overall chopping ratio is at least $98 \%$.

The LEBT chopper target (onto which the chopper electrodes deflect the beam) is designed to accept the full 150 Watts of beam power that would arise from having the whole $1 \mathrm{~ms}$ pulse land on the target at $60 \mathrm{~Hz}$. The heat load on the MEBT chopper target under cutoff conditions is no more than that during normal operations, so it is possible to use the combination of choppers to achieve full (better 
A, $-\mathrm{C}$

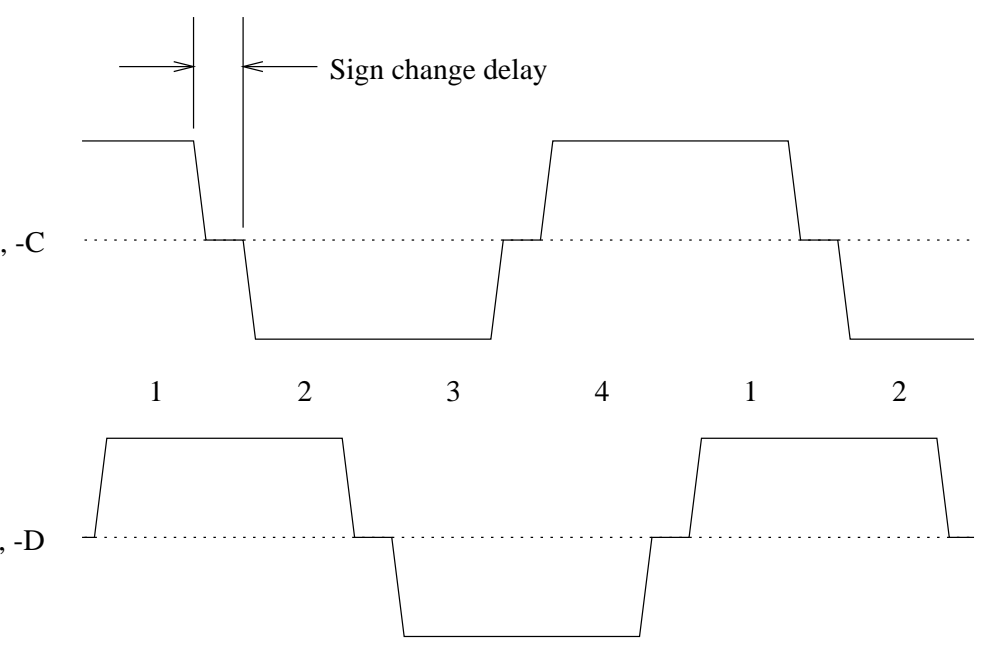

B, -D

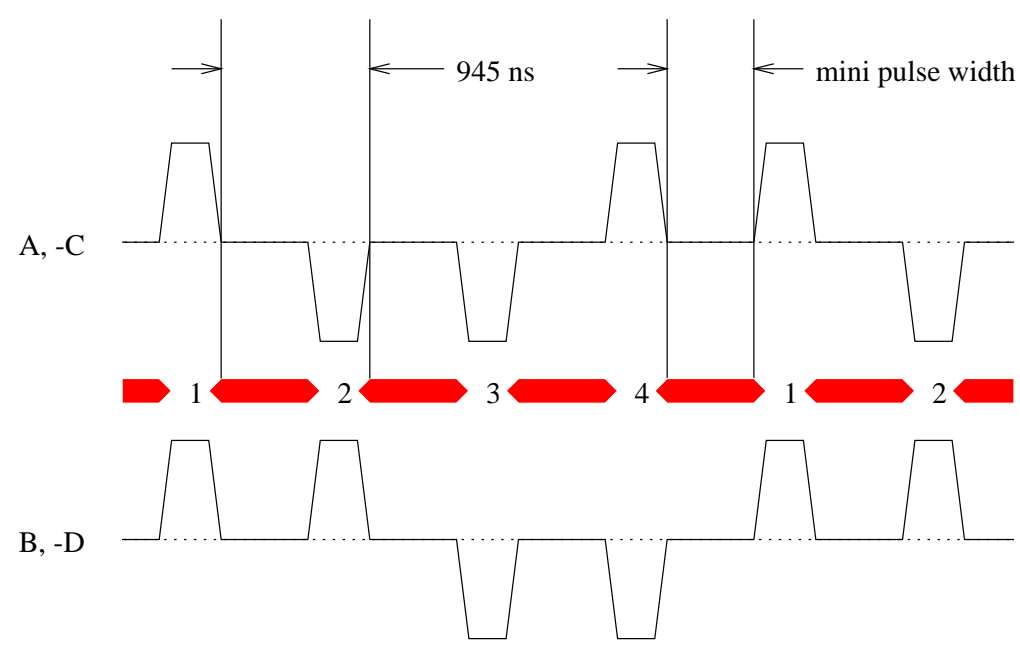

Beam Blanked

A
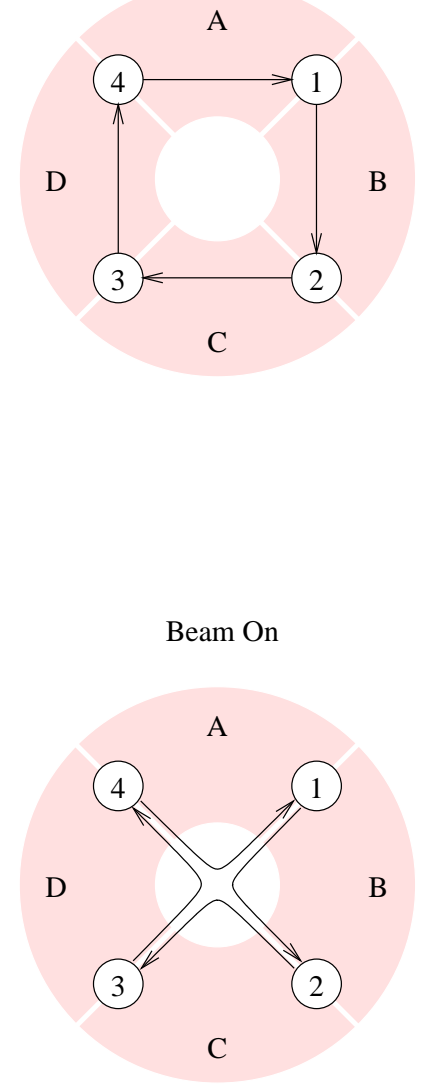

Figure 3: LEBT chopper waveforms at the mini-pulse scale.

than $10^{-4}$ ) extinction of the beam in the linac using the choppers, while leaving all RF systems running.

\section{MPS INTERACTION}

The Chopper controls need to be able to shift to beam blanking mode with microsecond latency, so the FPGA that controls these waveforms must have a direct connection from the MPS. The fault detection subsystems also need such a direct connection. Finally, the fault detection subsystems need to be able to communicate back to the MPS, with a similar low latency, so that backup shutoff can be triggered if this method fails. Additional levels of protection will be incorporated into the MPS. Current transformers on the $65 \mathrm{kV}$ power supply and in the MEBT will be used for verification of the chopper circuits.

\section{REFERENCES}

[1] J. Staples, private communication.

[2] C. Sibley, "Machine Protection System Requirements Document," Internal SNS Document.

[3] R. Shafer, "How Long a SNS Beam Pulse would Damage a Copper Accelerating Structure?," private correspondence.

[4] M. Ross et al., “Single Pulse Damage in Copper," Linac 2000 Proceedings, p. 47 (2000).

[5] J.W. Staples et al., "The SNS Four-Phase LEBT Chopper," Proceedings of the 1999 Particle Accelerator Conference, New York, 1999. 